\title{
A Four-Thirds law for phase randomization of stochastically perturbed oscillators and related phenomena
}

\author{
Robert Cogburn, James A. Ellison*
}

Department of Mathematics and Statistics, The University of New Mexico, Albuquerque, New Mexico 87131, USA. Email: ellison@math.unm.edu

Received: 19 May 1993/in revised form: 11 March 1994

\begin{abstract}
Let $I$ be a set of invariants and $\theta$ be a set of angle variables for a system of differential equations with an $O(\varepsilon)$ vector field. When time dependent stochastic perturbations, also of $O(\varepsilon)$, are added to the system, we have shown that under suitable conditions $I$ becomes a stochastic adiabatic invariant satisfying a diffusion equation on time scales of order $1 / \varepsilon^{2}$, in the limit as $\varepsilon \rightarrow 0$. Here we show that the angle variables converge weakly to a Gaussian Markov process on an $O\left(\varepsilon^{-4 / 3}\right)$ time scale, and thus the phase becomes randomized at these times. Application to nearly integrable Hamiltonian systems is considered.
\end{abstract}

\section{Introduction}

We consider the behavior of the stochastic differential equation in $\mathbb{R}^{d}$,

$$
\dot{x}=\varepsilon f(x, t)+\varepsilon F(x, t, \omega)+o\left(\varepsilon^{5 / 3}\right)
$$

as $\varepsilon \rightarrow 0$. We require that the expectation $E F(x, t)=0$ and that the time average

exists for all $x$.

$$
\bar{f}(x)=\lim _{t \rightarrow \infty} \frac{1}{t} \int_{0}^{t} f(x, s) d s
$$

Making the change of scale $v=\varepsilon t,(0.1)$ becomes

$$
\frac{d x}{d v}=f\left(x, \frac{v}{\varepsilon}\right)+F\left(x, \frac{v}{\varepsilon}, \omega\right)+o\left(\varepsilon^{2 / 3}\right) .
$$

Then (0.2) and the law of large numbers applied to $F$ suggest that the method of averaging may apply to $(0.3)$, and for small $\varepsilon$ the solution should be close to the "unperturbed equation"

$$
\frac{d x}{d v}=\bar{f}(x) .
$$

\footnotetext{
* Supported by NSF grant DMR-8704348
} 
In fact Khas'minskii [9] established that this was the case under suitable regularity conditions. Now suppose $y=I(x)$ taking values in $\mathbb{R}^{p}$ is an invariant for the unperturbed system:

$$
\frac{\partial I}{\partial x}(x) \bar{f}(x)=0 .
$$

Letting $X_{\varepsilon}$ denote a solution to $(0.1)$ and $Y_{\varepsilon}(t)=I\left(X_{\varepsilon}(t)\right)$, where $I$ is a smooth function, $Y_{\varepsilon}$ solves

$$
\frac{d Y_{\varepsilon}}{d t}=\varepsilon \frac{\partial I}{\partial x}\left(X_{\varepsilon}(t)\right) f\left(X_{\varepsilon}(t), t\right)+\varepsilon G\left(X_{\varepsilon}(t), t, \omega\right)+o\left(\varepsilon^{5 / 3}\right),
$$

where $G=\left(\frac{\partial I}{\partial x}\right) F$. In [3] we show that, provided the unperturbed system (0.4) is ergodic on the surfaces $I(x)=$ constant and certain regularity conditions apply, the $Y_{\varepsilon}(t)$ processes converge weakly to a diffusion on $O\left(\varepsilon^{-2}\right)$ time scales as $\varepsilon \rightarrow 0$.

In this study we suppose that, in addition to $X_{\varepsilon}(t)$ and $Y_{\varepsilon}(t)$, there is a third process $Z_{\varepsilon}(t)$ with values in $\mathbb{R}^{q}$ solving

$$
\frac{d Z_{\varepsilon}}{d t}=\varepsilon\left\{v\left(Y_{\varepsilon}(t)\right)+h\left(X_{\varepsilon}(t), t\right)+H\left(X_{\varepsilon}(t), t, \omega\right)\right\}+o\left(\varepsilon^{4 / 3}\right),
$$

where $E H(x, t)=0$ and $h$ has time average 0 .

The corresponding unperturbed system for $Z_{\varepsilon}$ is

$$
\frac{d z}{d t}=\varepsilon v(y),
$$

where $y$ is constant, hence the unperturbed $z(t)$ is a linear function of $t$.

The most obvious example fitting this description is an oscillator in phase space $x$, where $y$ is the energy and $z$ is the phase position. Or alternatively, $y$ and $z$ may be the "action" and "angle" variables of the system. This problem will be discussed in Sect. 2. In such cases there is a constant vector $\zeta$ whose $i^{\text {th }}$ coordinate $\zeta_{i}$ is the period of the $i^{\text {th }}$ coordinate $z_{i}$ of $z$. Taking $z(\bmod \zeta)$ to be the vector whose $i^{\text {th }}$ coordinate is $z_{i}\left(\bmod \zeta_{i}\right)$, from one point of view we should have $z(\bmod \zeta)=\Phi(x)$ for some function $\Phi: \mathbb{R}^{d} \rightarrow \mathbb{R}^{q}$, however this implies discontinuities in the $\Phi$ function, so in the usual way we regard $\Phi(x)$ to be a multivalued function, the branch in effect at any given time being determined by continuity.

Another possibility would be where $x$ is a laminar flow with shear, $y$ indexes the layer of the unperturbed flow and $z$ is the distance traveled in the layer. In both of these examples, using a suitable interpretation, we have $z=\Phi(x)$ and the unperturbed orbital derivative of $\Phi$ is

$$
\frac{\partial \Phi}{\partial x} \bar{f}(x)=v(y),
$$

a function of $y$, and (0.7) becomes

$$
\begin{aligned}
\frac{d Z_{\varepsilon}}{d t}= & \varepsilon\left\{v\left(Y_{\varepsilon}(t)\right)+\frac{\partial \Phi}{\partial x}\left(X_{\varepsilon}(t)\right)\left(\left(f\left(X_{\varepsilon}(t), t\right)-\bar{f}\left(X_{\varepsilon}(t)\right)+F\left(X_{\varepsilon}(t), t, \omega\right)\right)\right\}\right. \\
& +O\left(\varepsilon^{4 / 3}\right) .
\end{aligned}
$$


Let $x_{0}$ be the initial value for the $X_{\varepsilon}$ process and $y_{0}=I\left(x_{0}\right)$ and $z_{0}$ be corresponding initial values for the $Y_{\varepsilon}$ and $Z_{\varepsilon}$ processes. We will show that in the scaled time $\tau=\varepsilon^{4 / 3} t$, the process

$$
W_{\varepsilon}(\tau)=Z_{\varepsilon}\left(\tau \varepsilon^{-4 / 3}\right)-z_{0}-\tau \varepsilon^{-1 / 3} v\left(y_{0}\right)
$$

representing the deviation of $Z_{\varepsilon}$ from the unperturbed solution, converges weakly to a Gaussian Markov process as $\varepsilon \rightarrow 0$, under suitable conditions. From this we conclude, under a nondegeneracy condition, that $Z_{\varepsilon}(\bmod \zeta)$ becomes uniform on an $\varepsilon^{-4 / 3}$ time scale.

The following heuristic argument motivates the $\varepsilon^{-4 / 3}$ time scale. In a one degree of freedom nonlinear oscillator a point $x_{0}$ moves roughly on its phase plane oval with variance in action increasing like $E\left(J(t)-J_{0}\right)^{2}=\bar{\psi}\left(J_{0}\right) \varepsilon^{2} t$. The earliest time to expect uniformity on a thin energy shell containing $x_{0}$ would be when adjacent points on $J_{0}$ and $J(t)$ have separated by one revolution, i.e.,

$$
\left(v(J(t))-v\left(J_{0}\right)\right) \varepsilon t=2 \pi .
$$

Thus $v^{\prime}\left(J_{0}\right)\left(\bar{Y}\left(J_{0}\right) \varepsilon^{2} t\right)^{1 / 2} \varepsilon t \simeq 2 \pi$, which gives

$$
t \simeq\left(\frac{2 \pi}{v^{\prime}\left(J_{0}\right) \sqrt{\overline{\overline{4}}\left(J_{0}\right)}}\right)^{2 / 3} \varepsilon^{-4 / 3} .
$$

In Sect. 1 we introduce notation, discuss the assumptions and state the main results, and in Sect. 2 we discuss several examples that illustrate the theorems. Section 3 contains estimates, auxiliary results and proofs of the theorems.

\section{Formulation of the Main Results}

As in the introduction, the invariant $I: \mathbb{R}^{d} \rightarrow \mathbb{R}^{p}$. Since $y=I(x)$ varies only by small amounts on the time scales to be considered, it suffices to consider any open neighborhood $D_{1}$ of the initial value $y_{0}=I\left(x_{0}\right)$ in $\mathbb{R}^{p}$. We let $D_{0}$ be the largest connected set in $I^{-1}\left(D_{1}\right)$ such that $x_{0} \in D_{0}$.

In what follows $(\Omega, \mathscr{F}, P)$ is a probability space, and for each $x \in D_{0}$ and $t \geqq 0$, $F(x, t)=F(x, t, \omega)$ is an $\mathbb{R}^{p}$ valued random variable on $\Omega$.

For an $m \times n$ vector or matrix $M=\left(M_{j, k}\right)$, let $|M|=\sum_{j=1}^{m} \sum_{k=1}^{n}\left|M_{j, k}\right|$. When $M(x, s, t, \omega)$ is a vector or matrix valued function of $x_{0} \in D_{0}, s \geqq 0, t \geqq 0$ and $\omega \in \Omega$, let

$$
\begin{aligned}
& \|M\|=P-\text { ess sup } \sup _{\omega \in D_{0}} \sup _{s, t \geqq 0}|M(x, s, t, \omega)|,
\end{aligned}
$$

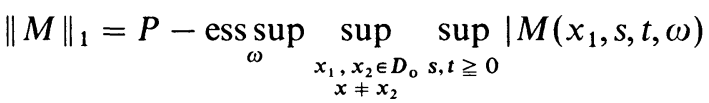

$$
\begin{aligned}
& -M\left(x_{2}, s, t, \omega\right)|/| x_{1}-x_{2} \mid+\|M\|, \\
& \|M\|_{2}=\max _{1 \leqq k \leqq d}\left\|\partial M / \partial x_{k}\right\|_{1}+\|M\|,
\end{aligned}
$$

and use the same conventions when $M$ depends on a subset of these arguments. Note that

$$
\|M\| \leqq\|M\|_{1} \leqq\|M\|_{2} .
$$

Now consider the assumptions: 
(A1) Writing (0.1) as the initial value problem

$$
\dot{x}=\varepsilon f(x, t)+\varepsilon F(x, t, \omega)+R(x, t, \omega, \varepsilon), x(0)=x_{0},
$$

we require $F$ and $R$ to be continuous in $x$ and $t, R$ to be locally $x$-Lipschitz and $\|F\|_{1}<\infty$. We also require $E F(x, t)=0$ for all $x, t$. All order statements for the limit as $\varepsilon \rightarrow 0$ are to be understood in terms of the first norm defined above, so the $o\left(\varepsilon^{5 / 3}\right)$ quantity in $(0.1)$ means $\|R\|=o\left(\varepsilon^{5 / 3}\right)$.

We require $f(x, t)$ to be almost periodic, with the Fourier representation $f(x, t)=\sum a_{k}(x) e^{i \lambda_{k} t}$. Hence the $\lambda_{k}$ are distnct, $\lambda_{-k}=-\lambda_{k}$ and $a_{-k}=a_{k}^{*}$ (the conjugate of $\left.a_{k}\right)$. Note that $a_{0}(x)=\bar{f}(x)$ as defined by (0.2). We assume

$$
\sum\left\|a_{k}\right\|<\infty, \sum_{k \neq 0}\left\|a_{k}\right\|_{1} /\left|\lambda_{k}\right|<\infty \text { and } \sum_{k \neq 0}\left\|a_{k}\right\|_{2} /\left|\lambda_{k}\right|^{1+0}<\infty
$$

for some $0<\theta \leqq 1$.

(A2) The mapping $I$ must satisfy $(0.5)$ and we require that $\left\|\frac{\partial I}{\partial x}\right\|_{2}<\infty$. Note that this assumption and (A1) imply that $\|G\|_{1}<\infty$.

(A3) The function $v: D_{1} \rightarrow \mathbb{R}^{q}$ must satisfy the global Lipschitz condition

$$
\left|\frac{\partial v}{\partial y}\left(y_{2}\right)-\frac{\partial v}{\partial y}\left(y_{1}\right)\right| \leqq C\left|y_{2}-y_{1}\right| \text {. }
$$

The equation (0.7) in $z$ must have continuous, locally $x$-Lipschitz right-hand side and we require $h$ to satisfy

$$
\sup _{x, t}\left|\int_{0}^{t} h(x, s) d s\right|<\infty,
$$

$\|h\|_{1}<\infty, E H(x, t)=0$ and $\|H\|_{1}<\infty$.

(A4) For $0 \leqq s \leqq t \leqq \infty$ let $\mathscr{F}_{s}^{t}$ be sub- $\sigma$-fields of $\mathscr{F}$ such that for $t_{1} \leqq t_{2} \leqq t_{3} \leqq t_{4}, \mathscr{F}_{t_{2}}^{t_{3}} \subset \mathscr{F}_{t_{1}}^{t_{4}}$ and such that $F(x, t)$ and $H(x, t)$ are $\mathscr{F}_{t}^{t}$ measurable for all $x \in D_{0}$ and $t \geqq 0$. If the initial state $x_{0}$ is random, then we also require $x_{0}$ to be $\mathscr{F}_{0}^{0}$ measurable. Let

$$
\rho(t)=\sup _{s \geqq 0} \sup _{A \in \mathscr{F}_{0}^{s}: P A>0} \sup _{B \in \mathscr{F}_{s+1}^{\infty}}|P(B \mid A)-P B| .
$$

We require the mixing condition

$$
\rho(t)=o\left(\frac{1}{t^{2}}\right)
$$

as $t \rightarrow \infty$.

Before stating the next assumption we need some notation. Let

$$
\Gamma(x, s, t):=E\left[F(x, s) F(x, t)^{T}\right]
$$

and

$$
\hat{\Gamma}(x, s):=\int_{s}^{\infty}(\Gamma(x, s, t)+\Gamma(x, t, s)) d t .
$$

An application of (A4) (see Lemma 3.1) shows that, for each fixed $s$ and $t,\|\Gamma(x, s, t)\|_{1} \leqq C \rho(|t-s|)$, and it follows easily that $\hat{\Gamma}$ is well defined 
and $\|\hat{\Gamma}\|_{1}<\infty$. Let

$$
\hat{\sharp}(x, t):=\frac{\partial I}{\partial x}(x) \hat{\Gamma}(x, t) \frac{\partial I}{\partial x}(x)^{T} .
$$

(A5) We assume there exists a function $Z(x)$ such that

$$
\sup _{x \in D_{0}, t \geqq 0}\left|\frac{1}{l} \int_{t}^{t+l} \hat{Z}(x, s) d s-Z(x)\right| \rightarrow 0
$$

as $l \rightarrow \infty$. Note $\|\hat{\Gamma}\|_{1}<\infty$ and (A2) imply $\|\hat{\psi}\|_{1}<\infty$ and $\|\not\|_{1}<\infty$.

(A6) Let $\bar{x}\left(t, x_{0}\right)$ be the solution to the initial value problem

$$
\dot{x}=\bar{f}(x), x(0)=x_{0} .
$$

Assume there exists a continuous function $\bar{Z}(y)$ of $y$ on $D_{1}$ such that

$$
\bar{Z}(I(x))=\lim _{l \rightarrow \infty} \frac{1}{l} \int_{0}^{t} Z(\bar{x}(t, x)) d t
$$

exists uniformly in $x$ in $D_{0}$.

(A6') Assume there is a matrix $M_{0}$ such that

$$
M_{0}=\lim _{l \rightarrow \infty} \frac{1}{l} \int_{0}^{l} Z(\bar{x}(t, x)) d t
$$

exists uniformly for $x \in I^{-1}\left\{y_{0}\right\}$, where $y_{0}$ is the initial value of the $Y_{\varepsilon}$ process, and assume that for any sequence $x_{n} \in D_{0}$ such that $I\left(x_{n}\right) \rightarrow y_{0}$, there exist $\hat{x}_{n} \in D_{0}$ such that $I\left(\hat{x}_{n}\right)=y_{0}$ and $\left|x_{n}-\hat{x}_{n}\right| \rightarrow 0$. Note that under (A6), $M_{0}=\overline{\bar{\psi}}\left(y_{0}\right)$.

Our first result describes the behavior of the $Y_{\varepsilon}(t)$ processes on $\varepsilon^{-4 / 3}$ time scales. Let $C_{k}[0, \infty)$ denote the space of continuous functions on $[0, \infty)$ to $\mathbb{R}^{k}$ with supremum norm.

Let

$$
V_{\varepsilon}(\tau):=\varepsilon^{-1 / 3}\left(Y_{\varepsilon}\left(\varepsilon^{-4 / 3} \tau\right)-y_{0}\right)
$$

Theorem 1.1. If assumptions (A1) through (A4) hold then the processes $\left\{V_{\varepsilon}(\tau)\right\}_{\tau} \geqq 0$ are relatively compact in $C_{p}[0, \infty)$ as $\varepsilon \rightarrow 0$. If, moreover, (A5) and either (A6) or $\left(\mathrm{A} 6^{\prime}\right)$ hold, then as $\varepsilon \rightarrow 0, V_{\varepsilon}(\tau)$ converges weakly to the Wiener process $V_{0}(\tau)$ having the representation

$$
V_{0}(\tau)=\left(\overline{4}\left(y_{0}\right)\right)^{1 / 2} B(\tau),
$$

where $B(\tau)$ is standard p-dimensional Brownian motion.

Remark. In [3] we show that $Y_{\varepsilon}\left(\tau / \varepsilon^{2}\right)$ converges weakly to $Y_{0}(\tau)$, where $d Y_{0}=\bar{\mu}\left(Y_{0}\right) d \tau+\bar{Z}\left(Y_{0}\right)^{1 / 2} d B(\tau)$. An heuristic perturbation argument at the $\varepsilon^{-4 / 3}$ time scale is consistent with (1.13).

Note. Here and in what follows, for a symmetric positive semidefinite (psd) matrix $M,(M)^{1 / 2}$ denotes its psd square root. Of course, the matrices $\hat{\Gamma}, \hat{\vec{\psi}}, \bar{\sharp}$, $\overline{4}$ are psd. 
Theorem 1.2. If assumptions (A1) through (A4) hold then the processes $\left\{W_{\varepsilon}(\tau)\right\}_{\tau} \geqq 0$ defined in (0.11) are relatively compact in $C_{q}[0, \infty)$ as $\varepsilon \rightarrow 0$. If, moreover, (A5) and either (A6) or $\left(\mathrm{A}^{\prime}\right)$ hold, then as $\varepsilon \rightarrow 0, W_{\varepsilon}(\tau)$ converges weakly to the Gaussian Markov process $W_{0}(\tau)=\frac{\partial y}{\partial y}\left(y_{0}\right)\left(\bar{Z}\left(y_{0}\right)\right)^{1 / 2} \int_{0}^{\tau} B(s) d s$, where $B(s)$ is standard p-dimensional Brownian motion. This q-dimensional process has continuous sample functions, zero mean and covariance for $0 \leqq \tau_{1} \leqq \tau_{2}$,

$$
E W_{0}\left(\tau_{1}\right) W_{0}\left(\tau_{2}\right)^{T}=\frac{\tau_{1}^{2}}{6}\left(3 \tau_{2}-\tau_{1}\right) \frac{\partial v}{\partial y}\left(y_{0}\right) \overline{\bar{Z}}\left(y_{0}\right) \frac{\partial v}{\partial y}\left(y_{0}\right)^{T} .
$$

When $\frac{\partial v}{\partial y}\left(y_{0}\right) \bar{Z}\left(y_{0}\right) \frac{\partial v}{\partial y}\left(y_{0}\right)^{T}$ is positive definite, it follows that $W_{0}(\tau)$ has a Gaussian distribution with large dispersion when $\tau$ is large, and this implies that $Z_{\varepsilon}\left(\tau \varepsilon^{-4 / 3}\right)(\bmod \zeta)$ is approximately uniform. In fact this "phase randomization" applies even without the mean stationarity and ergodic assumptions of (A5) and (A6) provided a minimal amount of stochastic perturbation is present, and this is the content of our third result.

For psd matrices $A, B$ we write $A \geqq B$ when $A-B$ is psd. We need:

(A7) Assume there is a $q \times q$ psd matrix function $\Delta(x), x \in D_{0}$, with $\|\Delta\|_{1}<\infty$ and that there is a constant positive definite matrix $\Delta_{0}$ and finite $T_{1}$ and $T_{2}$ such that

$$
\int_{t}^{t+T_{1}} \frac{\partial v}{\partial y}\left(y_{0}\right) \hat{\sharp}(x, s) \frac{\partial v}{\partial y}\left(y_{0}\right)^{T} d s \geqq \Delta(x)
$$

for all $t \geqq 0$, and

$$
\int_{0}^{T_{2}} \Delta(\bar{x}(t, x)) d t \geqq \Delta_{0}
$$

for all $x \in D_{0}$. Note that the time scales in (1.15) and (1.16) are different.

Theorem 1.3. If assumptions (A1) through (A4) and (A7) hold, then in the iterated limit as $\varepsilon \rightarrow 0$ followed by $\tau \rightarrow \infty, Z_{\varepsilon}\left(\tau \varepsilon^{-4 / 3}\right)(\bmod \zeta)$ converges in distribution to a uniform distribution on the rectangle $\left\{z: 0 \leqq z_{k}<\zeta_{k}, k=1, \ldots, q\right\}$, where $\zeta$ is an arbitrary element of $\mathbb{R}^{q}$ such that $\zeta_{k}>0, k=1, \ldots, q$.

Remarks. 1. The three theorems apply to the situation that $X_{\varepsilon}(0)=x_{0}$ fixed. If $X_{\varepsilon}(0)=X_{0}$ is a random variable and is $\mathscr{F}_{0}^{0}$ measurable, then Theorems 1.1 and 1.2 provide the conditional distributions of $V_{0}$ and $W_{0}$ given $X_{0}=x_{0}$, provided the appropriate regularity conditions hold for the given value of $x_{0}$, since (A4) implies asymptotic independence of $\left\{X_{\varepsilon}(t), t \geqq \delta\right\}$ from $X_{0}$ as $\varepsilon \rightarrow 0$ for each fixed $\delta>0$. Marginal distributions for the $V_{0}$ and $W_{0}$ processes are then obtained by integrating the conditional distributions with respect to the distribution of $X(0)$. In similar fashion Theorem 1.3 implies that the limit distribution of $Z_{\varepsilon}\left(\tau \varepsilon^{-4 / 3}\right)(\bmod \zeta)$ is still uniform.

2. Theorem 1.3 implies that an integrable Hamiltonian system with minimal stochastic perturbation (condition (A7)) asymptotically has a kind of ergodic averaging on constant energy surfaces, with this averaging taking place on time scales of order $\varepsilon^{-4 / 3}$. This result allows the extension of the adiabatic invariance results of [3] to this case under (A7), whether the unperturbed system is ergodic or not. We plan to provide the details in a subsequent paper. 


\section{Examples}

In this section we discuss examples which illustrate the theory.

Example $A$. In this example we will consider perturbations of the one degree of freedom nonlinear oscillator defined by the Hamiltonian

$$
H_{0}(x):=\frac{1}{2} x_{2}^{2}+U\left(x_{1}\right),
$$

where $U\left(x_{1}\right)$ is a symmetric bowl type potential so that all solutions are periodic. We write our example as

$$
\frac{d x}{d v}=\bar{f}(x)+p\left(x, \frac{v}{\varepsilon}\right)+F\left(x, \frac{v}{\varepsilon}, \omega\right) \quad x(0)=x_{0},
$$

where $\bar{f}(x)=\left(\begin{array}{c}x_{2} \\ -U^{\prime}\left(x_{1}\right)\end{array}\right), p(x, t)$ has zero $t$-mean and $E F=0$. If we let $\bar{x}\left(v, x_{0}\right)$ denote the solution of the unperturbed problem, then the transformation, $\left(x_{1}, x_{2}\right) \rightarrow(\theta, I)$, to the action angle variables of the unperturbed problem can be written

$$
x=\bar{x}(0 / v(I), \zeta(I)),
$$

where $v(I)$ is the frequency as a function of action associated with $H_{0}$ and $\zeta(I)$ is an appropriately chosen initial condition, on the closed integral curve for $H_{0}$ associated with $I$. If we let $y(t)=I(x(t))$ and $z(t)=\theta(x(t))$, then

$$
\begin{aligned}
& \dot{y}=\varepsilon I^{\prime}(x)[p(x, t)+F(x, t, \omega)], \quad y(0)=y_{0}=I\left(x_{0}\right), \\
& \dot{z}=\varepsilon[v(y)+D \theta(x)\{p(x, t)+F(x, t, \omega)\}], \quad z(0)=z_{0}=\theta\left(x_{0}\right) .
\end{aligned}
$$

We let $D_{1}$ be an open interval about $y_{0}=I\left(x_{0}\right)$ and thus $D_{0}=I^{-1}\left(D_{1}\right)$ is an energy shell about the initial energy oval, $\left\{x: H_{0}(x)=H_{0}\left(x_{0}\right)\right\}$. We assume the smoothness and almost periodicity conditions of (A1). For $U$ smooth, (A2) and (1.3) of (A3) are satisfied. To apply the theorems, we need to calculate $Z(x, t)$ and for illustration we assume

$$
F(x, t, \omega)=Q(x) \xi(t, \omega),
$$

where $\xi$ is a scalar such that $E \xi=0$ and that (A3) is satisfied. We define $\mathscr{F}_{s}^{t}=\sigma(\xi(\tau), t \leqq \tau \leqq s)$ and assume the mixing condition of (A4) is satisfied. Now recall that

$$
\begin{aligned}
V_{\varepsilon}(\tau) & :=\varepsilon^{-1 / 3}\left(Y_{\varepsilon}\left(\varepsilon^{-4 / 3} \tau\right)-y_{0}\right), \\
W_{\varepsilon}(\tau) & :=z_{\varepsilon}\left(\tau \varepsilon^{4 / 3}\right)-z_{0}-\tau \varepsilon^{-1 / 3} v\left(y_{0}\right),
\end{aligned}
$$

and at this point we know from Theorems 1.1 and 1.2 that $\left\{V_{\varepsilon}\right\}$ and $\left\{W_{\varepsilon}\right\}$ are relatively compact in their $C$-spaces. Condition (A6) is satisfied, so to proceed we have two options, depending on whether (A5) is satisfied or not. We look first at (A5).

Let $K(s, t)=E(\xi(s) \xi(t))$, then we have $\mid K\left(s+t, s \mid \leqq 2\|\xi(t)\|^{2} \rho(t)=0\left(1 / t^{2}\right)\right.$, where the inequality follows from Proposition 2.2, p. 346 of Ref. [6] and the equality is as $t \rightarrow \infty$ and follows from (A4). 
Clearly

$$
\Gamma(x, s, t)=Q(x) Q^{T}(x) K(s, t),
$$

and since $K(s, t)=K(t, s)$,

$$
\hat{\sharp}(x, t)=2 I^{\prime}(x) Q(x) Q^{T}(x) I^{\prime}(x) \int_{t}^{\infty} K(\tau, t) d \tau,
$$

which clearly exists because of our mixing condition. If

$$
\lim _{l \rightarrow \infty} \frac{1}{l} \int_{t}^{t+l} \int_{s}^{\infty} K(\tau, s) d \tau d s
$$

converges uniformly to a $t$-independent limit $\frac{1}{2} C$, then (A5) is satisfied with $\exists(x)=C I^{\prime}(x) Q(x) Q^{T}(x) I^{\prime}(x)^{T}$. A sufficient condition for this is the stationary of $\xi(t)$ in which case $C=2 \int_{0}^{\infty} K(s, 0) d s$. (A6) is automatically satisfied because $H_{0}$ is ergodic and

$$
\begin{aligned}
\bar{\sharp}(y)= & 2 \pi v(y) \frac{1}{\sqrt{2}} \int_{-a}^{a}\left[Z\left(x_{1}, \sqrt{2} \sqrt{h-U\left(x_{1}\right)}\right)\right. \\
& \left.+\not\left(-x_{1},-\sqrt{2} \sqrt{h-U\left(x_{1}\right)}\right)\right] \frac{d x_{1}}{\sqrt{h-U\left(x_{1}\right)}},
\end{aligned}
$$

where $h=h(y)$ and $a=a(y)$ are the energy and oscillation amplitude as a function of action, respectively, for the unperturbed motion.

Theorems 1.1 and 1.2 now yield

$$
V_{\varepsilon} \Rightarrow V_{0}, \text { where } V_{0}(\tau)=\bar{\zeta}\left(y_{0}\right)^{1 / 2} B(\tau),
$$

where $B(\tau)$ is standard Brownian motion, and

$$
W_{\varepsilon} \Rightarrow W_{0}, \quad \text { where } W_{0}(\tau)=v^{\prime}\left(y_{0}\right)^{1 / 2} \bar{Z}\left(y_{0}\right)^{1 / 2} \int_{0}^{\tau} B(s) d s .
$$

Thus, $W_{0}$ is Gauss-Markov with zero-mean and covariance given by (1.14).

Thus we obtain the phase randomization when $\overline{4}\left(y_{0}\right)>0$, which requires noise, and $v^{\prime}\left(y_{0}\right) \neq 0$, which requires $H_{0}$ to be a nonlinear oscillator. This type of phase randomization can also occur, in a coarse grained sense, without noise when the initial condition $y_{0}$ is not concentrated at a point [6].

In this case condition (A6) is automatic and (A5) holds, so there is no need to resort to condition (A7); nevertheless we discuss this condition to illustrate its use. Example B will illustrate its power.

Now assume there exists a $T_{1}$ such that

$$
\inf _{t \geqq 0} \int_{0}^{T_{1}} \int_{0}^{\infty} K(\tau+z+t, z+t) d \tau d z \geqq \alpha>0,
$$

then we can choose

$$
\Delta(x)=2 \alpha I^{\prime}(x) Q(x) Q(x)^{T} I^{\prime}(x)^{T}=2 \alpha v(y)^{-2}\left(U^{\prime}\left(x_{1}\right) Q_{1}+x_{2} Q_{2}\right)^{2} \geqq 0 .
$$

The second assumption in (A7) then amounts to $f(y)=$ $\max _{H_{0}(x)=h(y)}\left|U^{\prime}\left(x_{1}\right) Q_{1}(x)+x_{2} Q_{2}(\mathrm{x})\right|$ bounded away from zero on $D_{1}$ which is 
hardly any restriction at all. Theorem 1.3 then yields the phase randomization result.

Example B. Let $x=\left(\begin{array}{l}y \\ z\end{array}\right)$, where $y \in D_{1} \subset \mathbb{R}^{p}, z \in \mathbb{R}^{q}$ and $d=p+q$. Consider the IVP

$$
\begin{aligned}
& \frac{d y}{d t}=\varepsilon(g(x, t)+G(x, t, \omega)) \quad y(0)=y_{0}, \\
& \frac{d z}{d t}=\varepsilon(v(y)+h(x, t)+H(x, t, \omega)) \quad x(0)=x_{0},
\end{aligned}
$$

where $D_{1}$ is an open neighborhood of $y_{0}$ and all functions are $2 \pi$ periodic in $z, g$ and $h$ have zero $t$-mean and $E G=E H=0$. These equations fit our general framework with $f(x, t)=\left(\begin{array}{c}g(x, t) \\ v(y)+h(x, t)\end{array}\right), \bar{f}(x)=\left(\begin{array}{c}0 \\ v(y)\end{array}\right), F=\left(\begin{array}{c}G \\ H\end{array}\right), R=0$ and the invariant

$$
I(x)=\left(x_{1}, \ldots, x_{p}\right)^{T} .
$$

We assume the smoothness and almost periodicity conditions of (A1) and (A3). The fact that $D_{0}=D_{1} \times \mathbb{R}^{q}$ is unbounded is not a problem since all functions are $2 \pi$ periodic in $z$. (A2) is trivially satisfied and we assume the measureability and mixing conditions of (A4). It is easy to check that

$$
\hat{\sharp}(x, t)=\int_{s}^{\infty} E\left(G(x, s) G^{T}(x, t)\right)^{Q} d t
$$

and thus we see that our results are independent of whether $H=0$ or not. That is, it is the noise in $y$ that moves the system away from $y_{0}$ and allows the phase randomization due to the $v(y)$ term. In fact $g$ and $h$ do not affect the result either. If we assume that $G$ is stationary with $E\left(G(x, s) G^{T}(x, t)\right)=: C(x, s-t)$, then

$$
\hat{Z}(x, t)=\int_{-\infty}^{\infty} C(x, s) d s=: Z(x),
$$

and (A5) is satisfied.

In this example $\bar{x}$ is particularly simple,

$$
\bar{x}\left(t, x_{0}\right)=\left(\begin{array}{c}
y_{0} \\
v\left(y_{0}\right) t+z_{0}
\end{array}\right),
$$

and thus $Z\left(\bar{x}\left(t, x_{0}\right)\right)$ is quasi-periodic in $t$ since $\bar{Z}(x)$ is a $2 \pi$ periodic function of $z$. For $q \geqq 2$, (A6) is too restrictive since it will not be satisfied unless $v$ is a constant with rationally independent components, in whic case $v^{\prime}=0$ and there will be no phase randomization, however $\left(\mathrm{A}^{\prime}\right)$ may be satisfied. Now $I^{-1}\left(y_{0}\right)=\left\{\left(\begin{array}{c}y_{0} \\ z\end{array}\right)\left|z \in \mathbb{R}^{q}\right|\right.$ and the limit in (1.11) will exist uniformly in $I^{-1}\left(y_{0}\right)$ if the components of $v\left(y_{0}\right)$ are rationally independent (i.e. the rate of ergodization on the $q$-torus is independent of initial position on the torus). Let $x_{n}=\left(\begin{array}{l}y_{n} \\ z_{n}\end{array}\right)$ with $y_{n} \rightarrow y_{0}$ and $\hat{x}_{n}=\left(\begin{array}{c}y_{0} \\ z_{n}\end{array}\right)$, then $I\left(\hat{x}_{n}\right)=y_{0}$ and $\left|x_{n}-\hat{x}_{n}\right|=\left|y_{n}-y_{0}\right| \rightarrow 0$. Thus $\left(\mathrm{A}^{\prime}\right)$ is satisfied for $y_{0}$ such that the components of $v\left(y_{0}\right)$ are rationally independent and we can apply Theorems (1.1) and (1.2) to obtain the weak convergence of $\left\{V_{\varepsilon}\right\}$ and $\left\{W_{\varepsilon}\right\}$. The phase randomization follows if $v^{\prime}\left(y_{0}\right) \bar{y}\left(y_{0}\right) v^{\prime}\left(y_{0}\right)^{T}$ is positive definite (pd) and this is true if $v^{\prime}\left(y_{0}\right) Z\left(y_{0}\right) v^{\prime}\left(y_{0}\right)^{T}$ is pd. The latter is true if $Z(x)$ is positive definite, and the columns of $v^{\prime}\left(y_{0}\right)^{T}$ are linearly independent (which requires $q \leqq p$ ). 
However, since $v$ is non-constant and continuous we generally expect $y$ 's arbitrarily close to $y_{0}$ such that the components of $v(y)$ are rationally dependent and yet it seems that the phase randomization should not be so sensitive to $y_{0}$ particularly in the presence of noise. If we take $T_{1}=1$ and $\Delta(x)=v^{\prime}\left(y_{0}\right) \not$ $\left(x_{0}\right) v^{\prime}\left(y_{0}\right)^{T}$ and assume as before that this is positive definite, then the left-hand side of (1.16) is positive definite for all $T_{2}>0$ and Theorem 1.3 entails the phase randomization without (A5) and (A6').

Example C. Here we simply point out an important special case of Example B, namely a perturbation of an integral Hamiltonian system, $H_{0}$,

$$
H(J, \theta, t)=\varepsilon\left(H_{0}(J)+H_{d}(J, 0, t)+H_{r}(J, 0, t, \omega)\right),
$$

where $J \in \mathbb{R}^{n}, \theta \in T^{n}, H_{d}$ has zero time mean and $H_{r}$ zero expected value. The Hamiltonian equations of motion are now in the form of (2.6) with $y=J, z=\theta$, $\dot{y}=-\frac{\partial H}{\partial 0}$ and $\dot{z}=\frac{\partial H}{\partial J}$.

\section{Preliminary Estimates}

We assume (A1) through (A4) hold in all that follows. When (A5), (A6), (A6') or (A7) is used, this will be explicitly stated.

The following standard mixing result (e.g., see [10]) is needed:

Lemma 3.1. Let $\Xi(x, \omega)$ be an $\mathscr{F}_{s+t}^{\infty}$ measurable random variable with values in $\mathbb{R}^{p}$ and be Borel measurable in $x$ for each $\omega$. Let $\|\Xi\|<\infty$ and let $\xi(x)=E \Xi(x)$. Then for any $\mathscr{F}_{0}^{s}$ measurable random variable $Z$ with values in $\mathbb{R}^{p}$,

$$
\left\|E^{(s)} \Xi(Z)-\xi(Z)\right\| \leqq 2\|\Xi\| \rho(t) .
$$

In addition we need the following result (see Proposition 3.1 of [3]) where it is stated for a different time scaling):

Lemma 3.2. For each $\varepsilon_{0}>0$ there is a $C<\infty$ such that for all $0<\varepsilon \leqq \varepsilon_{0}$ and $t \geqq 0$,

$$
\left\|\varepsilon \int_{0}^{t} \frac{\partial I}{\partial x}\left(X_{\varepsilon}(s)\right) f\left(X_{\varepsilon}(s), s\right) d s\right\| \leqq C \varepsilon(\varepsilon t+1) .
$$

Lemma 3.3. For $t \geqq \varepsilon^{-1 / 2}$ there is a $C<\infty$ such that

$$
\left\|\int_{0}^{t} h\left(X_{\varepsilon}(s), s\right) d s\right\| \leqq C t \varepsilon^{1 / 2} .
$$

Proof. By (A3), $\|h\|_{1}<\infty$, and by (A1),

$$
\left|X_{\varepsilon}(r)-X_{\varepsilon}(s)\right| \leqq C_{0} \varepsilon|r-s|
$$

for some $C_{0}<\infty$. Hence

by (1.4).

$$
\begin{aligned}
\int_{0}^{t} h\left(X_{\varepsilon}(s), s\right) d s & =\int_{\varepsilon^{-1 / 2}}^{t} \varepsilon^{1 / 2} \int_{s-\varepsilon^{-1 / 2}}^{s} h\left(X_{\varepsilon}(r), s\right) d r d s+t 0\left(\varepsilon^{1 / 2}\right) \\
& =\varepsilon^{1 / 2} \int_{0}^{t} \int_{\max \left\{r, \varepsilon^{-1 / 2}\right\}}^{\min \left\{r+\varepsilon^{-1 / 2}, t^{2}\right\}} h\left(X_{\varepsilon}(r), s\right) d s d r+t 0\left(\varepsilon^{1 / 2}\right)=t 0\left(\varepsilon^{1 / 2}\right)
\end{aligned}
$$


Now let $L=L(\varepsilon)$ be chosen so $L(\varepsilon)=o\left(\varepsilon^{-1 / 3}\right)$ and $\rho(L(\varepsilon))=o\left(\varepsilon^{2 / 3}\right)$. This is possible since $\rho(t)=o\left(t^{-2}\right)$ by (A4).

Lemma 3.4. For $0 \leqq t_{1}<t_{2} \leqq \tau \varepsilon^{-4 / 3}$ and each fixed $\tau$, as $\varepsilon \rightarrow 0$,

$$
Y_{\varepsilon}\left(t_{2}\right)-Y_{\varepsilon}\left(t_{1}\right)=\varepsilon \int_{t_{1}}^{t_{2}} G\left(X_{\varepsilon}(t), t+L\right) d t+o\left(\varepsilon^{1 / 3}\right)
$$

and

$$
Z_{\varepsilon}\left(t_{2}\right)-Z_{\varepsilon}\left(t_{1}\right)=\varepsilon \int_{t_{1}}^{t_{2}} v\left(Y_{\varepsilon}(t)\right) d t+\varepsilon \int_{t_{1}}^{t_{2}} H\left(X_{\varepsilon}(t), t+L\right) d t+o(1) .
$$

Proof. Taking Lemma 3.2 into account, we have from (0.6) that

$$
Y_{\varepsilon}\left(t_{2}\right)-Y_{\varepsilon}\left(t_{1}\right)=\varepsilon \int_{t_{1}}^{t_{2}} G\left(X_{\varepsilon}(t), t\right) d t+o\left(\varepsilon^{1 / 3}\right)
$$

and

$$
\begin{aligned}
& \left|\varepsilon \int_{t_{1}}^{t_{2}} G\left(X_{\varepsilon}(t), t+L\right) d t-\varepsilon \int_{t_{1}}^{t_{2}} G\left(X_{\varepsilon}(t), t\right) d t\right| \\
& \leqq \varepsilon \int_{t_{1}+L}^{t_{2}+L} \mid G\left(X_{\varepsilon}(t-L), t\right)-G\left(X_{\varepsilon}(t), t\right) d t+0(\varepsilon L)=o\left(\varepsilon^{1 / 3}\right)
\end{aligned}
$$

by (3.4) and since $\|G\|_{1}<\infty$. This proves (3.5) and (3.6) follows by Lemma 3.3 and a similar argument, the $o(1)$ term arising from the defining $Z$ equation $(0.7)$.

Since $\left\|E^{(t)} G\left(X_{\varepsilon}(t), t+L\right)\right\| \leq\|G\| \rho(L)=o\left(\varepsilon^{2 / 3}\right)$ by Lemma 3.1, an immediate consequence of (3.5) is

Lemma 3.5. For $0 \leqq t_{1}<t_{2} \leqq \tau \varepsilon^{-4 / 3}$ and each fixed $\tau$,

$$
\left.\| E^{\left(t_{1}\right)} Y_{\varepsilon}\left(t_{2}\right)-Y_{\varepsilon}\left(t_{1}\right)\right) \|=o\left(\varepsilon^{1 / 3}\right),
$$

and, for $0 \leqq \tau_{1}<\tau_{2} \leqq \tau_{0}$ and each fixed $\tau_{0}$,

$$
\left\|E^{\left(\tau_{11} \varepsilon^{-4 / 3}\right)}\left(V_{\varepsilon}\left(\tau_{2}\right)-V_{\varepsilon}\left(\tau_{1}\right)\right)\right\| \rightarrow 0
$$

as $\varepsilon \rightarrow 0$.

Formula (3.5) also implies that $V_{\varepsilon}(\tau)=\hat{V}_{\varepsilon}(\tau)+o(1)$, where

$$
\hat{V}_{\varepsilon}(\tau):=\varepsilon^{2 / 3} \int_{0}^{\tau \varepsilon^{-4 / 3}} G\left(X_{\varepsilon}(t), t+L\right) d t,
$$

and we will establish Theorem 1.1 for $\hat{V}_{\varepsilon}(\tau)$, since the necessary estimates can be provided directly for this quantity.

To simplify notation in what follows we will use the following special symbols: for a vector or matrix $M, M^{Q}:=M M^{T}$ and for a square matrix $M, M^{S}:=M+M^{T}$. Note that if $M$ has $m$ rows and $n$ columns then $\left|M^{Q}\right| \leqq|M|^{2} \leqq m n\left|M^{Q}\right|$.

Lemma 3.6. For $0 \leqq t_{1}<t_{2} \leqq \tau \varepsilon^{-4 / 3}$ and any fixed $\tau$, there is $a C<\infty$ such that

$$
\left\|E^{\left(t_{1}\right)}\left(\int_{t_{1}}^{t_{2}} G\left(X_{\varepsilon}(t), t+L\right) d t\right)^{Q}\right\| \leqq C\left(t_{2}-t_{1}\right)
$$


and

$$
\left\|E^{\left(t_{1}\right)}\left(\int_{t_{1}}^{t_{2}} H\left(X_{\varepsilon}(t), t+L\right) d t\right)^{Q}\right\| \leqq C\left(t_{2}-t_{1}\right)
$$

Proof. Let

$$
m_{\varepsilon}(t)=\max _{0 \leqq t_{2}-t_{1} \leqq t}\left\|E^{\left(t_{1}\right)}\left(\int_{t_{1}}^{t_{2}} G\left(X_{\varepsilon}(s), s+L\right) d s\right)^{Q}\right\| .
$$

We use the decomposition

$$
\begin{aligned}
E^{\left(t_{1}\right)}\left(\int_{t_{1}}^{t_{2}} G\left(X_{\varepsilon}(t), t+L\right) d t\right)^{Q} & \\
= & E^{\left(t_{1}\right)}\left(\int_{t_{1}}^{t_{2}} G\left(X_{\varepsilon}(t), t+L\right) \int_{t}^{t+L} G\left(X_{\varepsilon}(s), s+L\right)^{T} d s d t\right)^{S} \\
& +E^{\left(t_{1}\right)}\left(\int_{t_{1}}^{t_{2}} G\left(X_{\varepsilon}(t), t+L\right) \int_{t+L}^{t_{2}} G\left(X_{\varepsilon}(s), s+L\right)^{T} d s d t\right)^{S} \\
= & : I_{1}+I_{2} .
\end{aligned}
$$

Applying Lemma 3.1,

$$
\left\|I_{1}\right\| \leqq\left\|E^{\left(t_{1}\right)}\left(\int_{t_{1}}^{t_{2}} G\left(X_{\varepsilon}(t), t+L\right) \int_{t}^{t+L} E^{(t+L)} G\left(X_{\varepsilon}(s), s+L\right)^{T} d s d t\right)^{S}\right\| \leqq C\left(t_{2}-t_{1}\right)
$$

and

$$
\begin{aligned}
\left\|I_{2}\right\| & \leqq 2\left\|E^{\left(t_{1}\right)} \int_{t_{1}+L}^{t_{2}+L}\left|E^{(s)} G\left(X_{\varepsilon}(s), s+L\right)\right|\left|\int_{t_{1}}^{s-L} G\left(X_{\varepsilon}(t), t+L\right)^{T} d t\right| d s\right\| \mid \\
& \leqq C_{2} \int_{t_{1}+L}^{t_{2}+L} \rho(L)\left|E^{\left(t_{1}\right)}\left(\int_{t_{1}}^{s-L} G\left(X_{\varepsilon}(t), t+L\right) d t\right)^{Q}\right|^{1 / 2} d s \\
& \leqq C_{2}\left(t_{2}-t_{1}\right) m_{\varepsilon}^{1 / 2}\left(t_{2}-t_{1}\right) o\left(\varepsilon^{2 / 3}\right)
\end{aligned}
$$

Thus

$$
m_{\varepsilon}(t) \leqq C_{1} t+C_{2} t m_{\varepsilon}^{1 / 2}(t) o\left(\varepsilon^{2 / 3}\right),
$$

and for $t \leqq \tau \varepsilon^{-4 / 3}$ it follows that $m_{\varepsilon} t \leqq C t$.

The second inequality is established by a similar argument.

Proposition 3.1. For $0 \leqq t_{1}<t_{2} \leqq \tau \varepsilon^{-4 / 3}$ and any fixed $\tau$, as $\varepsilon \rightarrow 0$,

$$
\left\|E^{\left(t_{1}\right)}\left(\int_{t_{1}}^{t_{2}} G\left(X_{\varepsilon}(t), t+L\right) d t\right)^{Q}-E^{\left(t_{1}\right)} \int_{t_{1}}^{t_{2}} \hat{\psi}\left(X_{\varepsilon}(t), t+L\right) d t\right\|=\left(t_{2}-t_{1}\right) o(1)
$$

and

$$
\left\|E^{\left(t_{1}\right)}\left(Y_{\varepsilon}\left(t_{2}\right)-Y_{\varepsilon}\left(t_{1}\right)\right)^{Q}-\varepsilon^{2} E^{\left(t_{1}\right)} \int_{t_{1}}^{t_{2}} \hat{\psi}\left(X_{\varepsilon}(t), t\right) d t\right\|=o\left(\varepsilon^{2 / 3}\right) .
$$


Moreover, if (A5) holds then

$$
\left\|E^{\left(t_{1}\right)}\left(Y_{\varepsilon}\left(t_{2}\right)-Y_{\varepsilon}\left(t_{1}\right)\right)^{Q}-\varepsilon^{2} E^{\left(t_{1}\right)} \int_{t_{1}}^{t_{2}} Z\left(X_{\varepsilon}(t)\right) d t\right\|=o\left(\varepsilon^{2 / 3}\right) .
$$

Proof. Apply the decomposition in (3.12). The term $I_{2}$ is $\left(t_{2}-t_{1}\right) o(1)$ by (3.13), and applying Lemma 3.1,

$$
\begin{aligned}
I_{1}= & E^{\left(t_{1}\right)}\left(\int_{t_{1}}^{t_{2}} \int_{t}^{t+L} E^{(t)}\left[G\left(X_{\varepsilon}(t), t+L\right) G\left(X_{\varepsilon}(s), s+L\right)^{T}\right] d s d t\right)^{S} \\
& +\left(t_{2}-t_{1}\right) O\left(\varepsilon L^{2}\right) \\
= & E^{\left(t_{1}\right)}\left(\int_{t_{1}}^{t_{2}} \int_{t}^{\infty} \frac{\partial I}{\partial x}\left(X_{\varepsilon}(t)\right) \Gamma\left(X_{\varepsilon}(t), t+L, s+L\right)^{S} \frac{\partial I}{\partial x}\left(X_{\varepsilon}(t)\right)^{T} d s d t\right. \\
& +\left(t_{2}-t_{1}\right) o(1) \\
= & E^{\left(t_{1}\right)} \int_{t_{1}}^{t_{2}} \hat{\psi}\left(X_{\varepsilon}(t), t+L\right) d t+\left(t_{1}-t_{2}\right) o(1)
\end{aligned}
$$

proving (3.14). It follows from (3.5) and (3.7) that

$$
E^{\left(t_{1}\right)}\left(Y_{\varepsilon}\left(t_{2}\right)-Y_{\varepsilon}\left(t_{1}\right)\right)^{Q}=\varepsilon^{2} E^{\left(t_{1}\right)}\left(\int_{t_{1}}^{t_{2}} G\left(X_{\varepsilon}(t), t+L\right) d t\right)^{Q}+o\left(\varepsilon^{2 / 3}\right),
$$

and (3.15) follows from this, (3.14) and since

$$
\left.\mid \hat{\sharp}\left(X_{\varepsilon}(t+L), t+L\right)\right)-\hat{\sharp}\left(X_{\varepsilon}(t), t+L\right) \mid \leqq \varepsilon C L .
$$

For (3.16) note that

$$
\begin{aligned}
\int_{t_{1}}^{t_{2}} \hat{\xi}\left(X_{\varepsilon}(t), t\right) d t & =\int_{t_{1}}^{t_{2}} \frac{1}{L} \int_{t}^{t+L} \hat{\xi}\left(X_{\varepsilon}(s), t\right) d s d t+\left(t_{2}-t_{1}\right) o(1) \\
& =\int_{t_{1}+L}^{t_{2}} \frac{1}{L} \int_{s-L}^{s} \hat{\sharp}\left(X_{\varepsilon}(s), t\right) d t d s+\left(t_{2}-t_{1}\right) o(1)+O(L) \\
& =\int_{t_{1}}^{t_{2}} \Downarrow\left(X_{\varepsilon}(s)\right) d s+o\left(\varepsilon^{-4 / 3}\right)
\end{aligned}
$$

by (A5).

Lemma 3.7. For each fixed $\tau$, as $\varepsilon \rightarrow 0$,

$$
E\left\{\max _{0 \leqq t \leqq \tau \varepsilon^{-4 / 3}}\left|Z_{\varepsilon}(t)-z_{0}-\varepsilon \int_{0}^{t} v\left(Y_{\varepsilon}(s)\right) d s\right|\right\} \rightarrow 0 .
$$

Proof. By (3.6), it suffices to show that

$$
B_{\varepsilon}:=E\left\{\max _{0 \leqq t \leqq \tau \varepsilon^{-4 / 3}}\left|\varepsilon \int_{0}^{t} H\left(X_{\varepsilon}(s), s+L\right) d s\right|\right\} \rightarrow 0 .
$$


as $\varepsilon \rightarrow 0$. Let $\lambda=\varepsilon^{-8 / 9}$. By (A1) and (A3), $\|H\|_{1}<\infty$ and, with $k$ an integer,

$$
\begin{aligned}
B_{\varepsilon} & \leqq E\left\{\max _{1 \leqq k \leqq \tau \varepsilon^{-4 / 3} / \lambda}\left|\varepsilon \int_{0}^{k \lambda} H\left(X_{\varepsilon}(s), s+L\right) d s\right|\right\}+O\left(\varepsilon^{1 / 9}\right) \\
& \leqq \sum_{k=1}^{\left[\tau \varepsilon^{-4 / 3 / \lambda]}\right.} E\left|\varepsilon \int_{(k-1) \lambda}^{k \lambda} H\left(X_{\varepsilon}(s), s+L\right) d s\right|+O\left(\varepsilon^{1 / 9}\right) \\
& \leqq C \sum_{k=1}^{\left[\tau \varepsilon^{-4 / 3} / \lambda\right]} \varepsilon\left|E\left(\int_{(k-1) \lambda}^{k \lambda} H\left(X_{\varepsilon}(s), s+L\right) d s\right)^{Q}\right|^{1 / 2}+O\left(\varepsilon^{1 / 9}\right) \\
& =O\left(\varepsilon^{1 / 9}\right)
\end{aligned}
$$

by (3.11).

Lemma 3.8. For each fixed $\tau$, as $\varepsilon \rightarrow 0$,

$$
E\left(\max _{0 \leqq t \leqq \tau \varepsilon^{-4 / 3}}\left|\varepsilon \int_{0}^{t} v\left(Y_{\varepsilon}(s)\right) d s-\varepsilon\left(t v\left(y_{0}\right)+\frac{\partial v}{\partial y}\left(y_{0}\right) \int_{0}^{t}\left(Y_{\varepsilon}(s)-y_{0}\right) d s\right)\right|\right\} \rightarrow 0 .
$$

Proof. By Taylor's theorem,

$$
\begin{aligned}
& v\left(Y_{\varepsilon}(s)\right)-v\left(y_{0}\right)-\frac{\partial v}{\partial y}\left(y_{0}\right)\left(Y_{\varepsilon}(s)-y_{0}\right), \\
& =\int_{0}^{1}\left[\frac{\partial v}{\partial y}\left(y_{0}+\eta\left(Y_{\varepsilon}(s)-y_{0}\right)\right)-\frac{\partial v}{\partial y}\left(y_{0}\right)\right] d \eta\left(Y_{\varepsilon}(s)-y_{0}\right) .
\end{aligned}
$$

Integrating this over $[0, t]$ and using (1.3) gives that the left-hand side of (3.18) is at most

$$
C \varepsilon E \int_{0}^{\tau \varepsilon^{-4 / 3}}\left|Y_{\varepsilon}(s)-y_{0}\right|^{2} d s \leqq C \varepsilon \int_{0}^{\tau \varepsilon^{-4 / 3}} E\left|\left(Y_{\varepsilon}(s)-y_{0}\right)^{Q}\right| d s
$$

for some constant $C$. However, there exists a constant $C_{1}>0$ such that $\left|E M^{Q}\right|$ $\geqq C_{1} E\left|M^{Q}\right|$, and thus using (3.15) and the boundedness of $\hat{4}$ gives that the last quantity is at most

$$
C_{2} \varepsilon \int_{0}^{\tau \varepsilon^{-4 / 3}}\left|E\left(Y_{\varepsilon}(s)-y_{0}\right)^{Q}\right| d s=o\left(\varepsilon^{1 / 3}\right)
$$

An immediate consequence of Lemma 3.7 and 3.8 is

Proposition 3.2. For each fixed $\tau_{0}$, as $\varepsilon \rightarrow 0$,

$$
E\left\{\max _{0 \leqq \tau \leqq \tau 0}\left|W_{\varepsilon}(\tau)-\varepsilon \frac{\partial \nu}{\partial y}\left(y_{0}\right) \int_{0}^{\tau \varepsilon^{-4 / 3}}\left(Y_{\varepsilon}(t)-y_{0}\right) d t\right|\right\} \rightarrow 0
$$

Based on this result and (3.9) it suffices to consider

$$
\hat{W}_{\varepsilon}(\tau):=\frac{\partial v}{\partial y}\left(y_{0}\right) \int_{0}^{\tau} \hat{V}_{\varepsilon}(s) d s .
$$

In what follows we establish Theorem 1.2 for $\hat{W}_{\varepsilon}(\tau)$. 
Lemma 3.9. For $0 \leqq \tau<\tau+\delta \leqq \tau_{0}$ and any fixed $\tau_{0}$, there is a constant $C$ depending only on $\tau_{0}$ such that, as $\varepsilon \rightarrow 0$,

$$
E\left|\hat{V}_{\varepsilon}(\tau+\delta)-\hat{V}_{\varepsilon}(\tau)\right|^{4} \leqq C \delta^{2}
$$

and

$$
E\left|\hat{W}_{\varepsilon}(\tau+\delta)-\hat{W}_{\varepsilon}(\tau)\right|^{4} \leqq C \delta^{2}
$$

Proof. 1. By (3.9),

$$
\left|\hat{V}_{\varepsilon}(\tau+\delta)-\hat{V}_{\varepsilon}(\tau)\right|^{4} \leqq C\left|\varepsilon^{2 / 3} \int_{\tau \varepsilon^{-4 / 3}}^{(\tau+\delta) \varepsilon^{-4 / 3}} G\left(X_{\varepsilon}(t), t+L\right) d t\right|^{4} .
$$

Let

$$
U_{\varepsilon, t}(l)=\int_{t}^{t+l} G\left(X_{\varepsilon}(s), s+L\right) d s
$$

and

$$
\beta(l)=\sup _{0 \leqq s \leqq l, 0<\varepsilon \leqq \varepsilon_{0}, t \geqq 0} E\left|U_{\varepsilon, t}(s)\right|^{4} .
$$

To simplify notation we treat $U=U_{\varepsilon, t}$ as one dimensional. Improvising on a method of Borodin [2],

$$
E U^{4}(l)=4 \int_{t}^{t+l} E G\left(X_{\varepsilon}(t+s), t+s+L\right) U^{3}(s) d s=4 \int_{t}^{t+l} \sum_{k=0}^{3} E I_{k}(s) d s,
$$

where

$$
I_{k}(s)=\left(\begin{array}{l}
3 \\
k
\end{array}\right) G\left(X_{\varepsilon}(t+s), t+s+L\right)(U(s)-U(s-L))^{k} U^{3-k}(s-L) .
$$

For $s \leqq l$,

$$
\left|E I_{0}(s)\right|=\left|E U^{3}(s-L) E^{(t+s)} G\left(X_{\varepsilon}(t+s), t+s+L\right)\right| \leqq C_{0} \beta^{3 / 4}(l) \varepsilon^{2 / 3},
$$

and for $k=1,2,3$,

$$
\begin{aligned}
\left|E I_{k}(s)\right| \leqq & k !\left(\begin{array}{l}
3 \\
k
\end{array}\right) \int_{t+s-L}^{t+s} d u_{1} \ldots \int_{t+s-L}^{u_{k-1}} d u_{k} \\
& \cdot\left|E U^{3-k}(s-L) \prod_{j=1}^{k} G\left(X_{\varepsilon}\left(u_{j}\right), u_{j}+L\right) E^{\left(u_{1}+L\right)} G\left(X_{\varepsilon}(t+s), t+s+L\right)\right| \\
\leqq & C_{k} \beta^{(3-k) / 4}(l) L^{k-1} .
\end{aligned}
$$

Combining these estimates yields

$$
\beta(l) \leqq C_{4} l\left(\beta^{3 / 4}(l) \varepsilon^{2 / 3}+\beta^{1 / 2}(l)+\beta^{1 / 4}(l) \varepsilon^{1 / 3}+\varepsilon^{2 / 3}\right) .
$$

Now suppose $1 \leqq l \leqq \tau_{0} \varepsilon^{-4 / 3}$ and set $B=B(l)=B(l) / l^{2}$. Then

$$
B l^{2} \leqq C_{5} l^{2}\left(B^{3 / 4}+B^{1 / 2}+B^{1 / 4}+1\right) .
$$

It follows that $B(l) \leqq C_{6}<\infty$, and applying this estimate to (3.23) yields the first assertion. 
2. For the second assertion use

$$
E\left|\hat{W}_{\varepsilon}(\tau+\delta)-\hat{W}_{\varepsilon}(\tau)\right|^{4} \leqq C_{1} E\left|\int_{\tau}^{\tau+\delta} \hat{V}_{\varepsilon}(s) d s\right|^{4} \leqq C_{1} \delta^{4} \varepsilon^{8 / 3} \beta\left(\tau_{0} \varepsilon^{-4 / 3}\right) \leqq C_{2} \delta^{2}
$$

In [3] we use the following variation on the first order stochastic averaging result of Khas'minskii (see $[9,8])$ :

Proposition 3.3. For each fixed $l$,

$$
\sup _{t}\left\|E^{(t)} \max _{0 \leqq s \leqq l}\left|X_{\varepsilon}\left(t+s \varepsilon^{-1}\right)-\bar{x}\left(s, X_{\varepsilon}(t)\right)\right|\right\| \rightarrow 0,
$$

as $\varepsilon \rightarrow 0$.

The result in [3] is stated for times scaled by $1 / \varepsilon^{2}$. As noted there, the uniformity in $t$ and the use of $E^{(t)}$ in place of $E$ are justified by uniformity of conditions (A1), (A2) and (A4).

Lemma 3.10. Let (A5) hold and let (A6) or (A6') hold. Then for each $\delta>0$, as $\varepsilon \rightarrow 0$,

$$
\left\|E^{\left(\tau \varepsilon^{-4 / 3}\right)}\left(\hat{V}_{\varepsilon}(\tau+\delta)-\hat{V}_{\varepsilon}(\tau)\right)^{Q}-\delta \bar{\psi}\left(y_{0}\right)\right\| \rightarrow 0 .
$$

(To simplify notation we use $\bar{\psi}\left(y_{0}\right)$ for $M_{0}$ under $\left(\mathrm{A} 6^{\prime}\right)$ as well as (A6) since as noted under $\left(\mathrm{A} 6^{\prime}\right), M_{0}$ and $\bar{Z}\left(y_{0}\right)$ coincide when (A6) and $\left(\mathrm{A}^{\prime}\right)$ both hold.

Proof. For fixed $\tau$ and $l$, using Proposition 3.3 at the second equality,

$$
\begin{aligned}
E^{\left(\tau \varepsilon^{-4 / 3}\right)} \frac{1}{\varepsilon^{1 / 3} l} \int_{\tau}^{\tau+\varepsilon^{1 / 3 l}} Z\left(X_{\varepsilon}\left(s \varepsilon^{-4 / 3}\right)\right) d s & =\frac{1}{l} \int_{0}^{l} E^{\left(\tau \varepsilon^{-4 / 3}\right)} Z\left(X_{\varepsilon}\left(\tau \varepsilon^{-4 / 3}+r \varepsilon^{-1}\right)\right) d r \\
& =\frac{1}{l} \int_{0}^{l} E^{\left(\tau \varepsilon^{-4 / 3}\right)} Z\left(\bar{x}\left(r, X_{\varepsilon}\left(\tau \varepsilon^{-4 / 3}\right)\right)\right) d r+o\left(\varepsilon^{1 / 3}\right) .
\end{aligned}
$$

Under (A6) the last integral is $E^{\left(\tau \varepsilon^{-4 / 3}\right)} \bar{Z}\left(Y_{\varepsilon}\left(\tau \varepsilon^{-4 / 3}\right)\right)+o(1)$ in the iterated limit as $\varepsilon \rightarrow 0$ then $l \rightarrow \infty$, which is $\bar{\sharp}\left(y_{0}\right)+o(1)$ since $\left\|E^{\left(\tau \varepsilon^{-4 / 3}\right)}\left(Y_{\varepsilon}\left(\tau \varepsilon^{-4 / 3}\right)-y_{0}\right)^{Q}\right\| \rightarrow 0$ as $\varepsilon \rightarrow 0$ by $(3.15)$ and since $\|\overline{4}\|_{1} \leqq\|Z\|_{1}<\infty$.

Under $\left(A 6^{\prime}\right)$, the above convergence of $Y_{\varepsilon}\left(\tau \varepsilon^{-4 / 3}\right)$ implies there exist $\hat{X}_{\varepsilon}$ such that $I\left(\hat{X}_{\varepsilon}\right)=y_{0}$ and $X_{\varepsilon}\left(\tau \varepsilon^{-4 / 3}\right)-\hat{X}_{\varepsilon} \rightarrow 0$ uniformly in $P^{\left(\tau \varepsilon^{-4 / 3}\right)}$ probability. Using the continuous dependence of $\bar{x}(r, x)$ on $x$ and the dominated convergence theorem, it again follows that

$$
E^{\left(\tau \varepsilon^{-4 / 3}\right)} \int_{\tau}^{\tau+\varepsilon^{1 / 3 \prime}} Z\left(X_{\varepsilon}\left(s \varepsilon^{-4 / 3}\right)\right) d s=\varepsilon^{1 / 3} l\left(\bar{\zeta}\left(y_{0}\right)+o(1)\right)
$$

as $\varepsilon \rightarrow 0$ then $l \rightarrow \infty$.

Applying (1.12), (3.7), (3.16) and a change of the integration variable,

$$
\begin{aligned}
E^{\left(\tau \varepsilon^{-4 / 3}\right)}\left(\hat{V}_{\varepsilon}(\tau+\delta)-\hat{V}_{\varepsilon}(\tau)\right)^{Q} & =\varepsilon^{-2 / 3} E^{\left(\tau \varepsilon^{-4 / 3}\right)}\left(Y_{\varepsilon}\left((\tau+\delta) \varepsilon^{-4 / 3}\right)-Y_{\varepsilon}\left(\tau \varepsilon^{-4 / 3}\right)\right)^{Q}+o(1) \\
& =E^{\left(\tau \varepsilon^{-4 / 3}\right)} \int_{\tau}^{\tau+\delta} Z\left(X_{\varepsilon}\left(\sigma \varepsilon^{-4 / 3}\right)\right) d \sigma+o(1)
\end{aligned}
$$




$$
\begin{aligned}
& =\int_{\tau}^{\tau+\delta} \frac{1}{\varepsilon^{1 / 3} l} \int_{\sigma}^{\sigma+\varepsilon^{1 / 3} l} E^{\left(\tau \varepsilon^{-4 / 3}\right)} Z\left(X_{\varepsilon}\left(s \varepsilon^{-4 / 3}\right)\right) d s d \sigma+o(1) \\
& =\int_{\tau}^{\tau+\delta}\left(\bar{Z}\left(y_{0}\right)+o(1)\right) d \sigma+o(1) \\
& =\delta \bar{Z}\left(y_{0}\right)+o(1),
\end{aligned}
$$

as $\varepsilon \rightarrow 0$ then $l \rightarrow \infty$.

Proof of Theorem 1.1. By (3.9) it suffices to prove the asserted limit for the processes $\left\{\hat{V}_{\varepsilon}(\tau), \tau \geqq 0\right\}$ as $\varepsilon \rightarrow 0$. Lemma 3.9 implies that these processes are relatively compact in $C_{p}\left[0, \tau_{0}\right)$ for each $\tau_{0}$, and this implies relative compactness in $C_{p}[0, \infty)([1][7])$. Moreover fourth moments are bounded on each $\left[0, \tau_{0}\right)$, so the $\hat{V}_{\varepsilon}(\tau)$ are uniformly integrable as $\varepsilon \rightarrow 0$.

To prove the asserted weak convergence when (A5) and (A6) hold, it suffices to show that for any weakly convergent sequence $\widehat{V}_{\varepsilon_{n}} \rightarrow V_{0}$ the limit process has the asserted distribution. Using the Skorohod representation theorem, we can assume without loss of generality that $\hat{V}_{\varepsilon_{n}} \rightarrow V_{0}$ a.s. Now let $\tau_{1}<\tau_{2}<\cdots<\tau_{k} \leqq \tau<\tau+\delta$ and $\eta: \mathbb{R}^{k} \rightarrow \mathbb{R}^{1}$ be bounded and continuous. Then, using (3.25) at the third equality below,

$$
\begin{aligned}
E & {\left[\eta\left(V_{0}\left(\tau_{1}\right), \ldots, V_{0}\left(\tau_{k}\right)\right)\left(V_{0}(\tau+\delta)-V_{0}(\tau)\right)^{Q}\right] } \\
& =\lim _{n \rightarrow \infty} E\left[\eta\left(\hat{V}_{\varepsilon_{n}}\left(\tau_{1}\right), \ldots, \hat{V}_{\varepsilon_{n}}\left(\tau_{k}\right)\right)\left(\hat{V}_{\varepsilon_{n}}(\tau+\delta)-\hat{V}_{\varepsilon_{n}}(\tau)\right)^{Q}\right] \\
& =\lim _{n \rightarrow \infty} E\left[\eta\left(\hat{V}_{\varepsilon_{n}}\left(\tau_{1}\right), \ldots, \hat{V}_{\varepsilon_{n}}\left(\tau_{k}\right)\right) E^{\left(\tau \varepsilon^{-4 / 3}\right)}\left(\hat{V}_{\varepsilon_{n}}(\tau+\delta)-\hat{V}_{\varepsilon_{n}}(\tau)\right)^{Q}\right] \\
& =\lim _{n \rightarrow \infty} E\left[\eta\left(\hat{V}_{\varepsilon_{n}}\left(\tau_{1}\right), \ldots, \hat{V}_{\varepsilon_{n}}\left(\tau_{k}\right)\right) \delta \bar{\psi}\left(y_{0}\right)\right] \\
& =E\left[\eta\left(V_{0}\left(\tau_{1}\right), \ldots, V_{0}\left(\tau_{k}\right)\right) \delta \bar{\psi}\left(y_{0}\right)\right] .
\end{aligned}
$$

Letting $\mathscr{A}_{\tau}=\sigma\left(V_{0}(s), s<\tau\right)$, it follows that

$$
E\left(\left(V_{0}(\tau+\delta)-V_{0}(\tau)\right)^{Q} \mid \mathscr{A}_{\tau}\right)=\delta \overline{\bar{Z}}\left(y_{0}\right) \text { a.s., }
$$

and a similar argument using (3.8) in place of (3.25) shows that $V_{0}(\tau)$ is a martingle. It then follows that $V_{0}(\tau)=\left(\bar{\zeta}\left(y_{0}\right)\right)^{1 / 2} B(\tau)$, where $B(\tau)$ is standard Browninan motion in $\mathbb{R}^{q}$. (The essential ideas are in [4] and a modern treatment of the multivariate case can be found in [7].)

Proof of Theorem 1.2. The relative compactness in $C_{q}\left[0, \tau_{0}\right]$ for each $\tau_{0}$ follows from (3.22).

Now assume (A5) and (A6), so $\hat{V}_{\varepsilon} \rightarrow V_{0}$ weakly as $\varepsilon \rightarrow 0$. Since by (3.20), each $\hat{W}_{\varepsilon}(\tau)$ is a continuous functional of $\left\{\hat{V}_{\varepsilon}(s): s \leqq \tau\right\}$, it follows that $\hat{W}_{\varepsilon}$ converges weakly to $W_{0}$, where the process $\left\{W_{0}(\tau): \tau \geqq 0\right\}$ has the distribution of

$$
\frac{\partial v}{\partial y}\left(y_{0}\right) \int_{0}^{\tau} V_{0}(s) d s, \tau>0 .
$$


Since $V_{0}$ is Gaussian, it follows that $W_{0}$ is Gaussian, too, and the independence of the increments of $V_{0}$ implies $W_{0}$ is a Markov process. Moreover

and for $0 \leqq \tau_{1} \leqq \tau_{2}$

$$
E W_{0}(\tau)=\frac{\partial v}{\partial y}\left(y_{0}\right) \int_{0}^{\tau} E V_{0}(s) d s=0,
$$

$$
\begin{aligned}
E\left[W_{0}\left(\tau_{1}\right) W_{0}\left(\tau_{2}\right)^{T}\right] & =\frac{\partial v}{\partial y}\left(y_{0}\right) \int_{0}^{\tau_{1}} \int_{0}^{\tau_{2}} E\left[V_{0}(s) V_{0}(t)^{T}\right] d t d s \frac{\partial v}{\partial y}\left(y_{0}\right)^{T} \\
& =\frac{\partial v}{\partial y}\left(y_{0}\right) \overline{4}\left(y_{0}\right) \frac{\partial v}{\partial y}\left(y_{0}\right)^{T} \int_{0}^{\tau_{1}} \int_{0}^{\tau_{2}} \min \{s, t\} d t d s \\
& =\frac{\tau_{1}^{2}}{6}\left(3 \tau_{2}-\tau_{1}\right) \frac{\partial v}{\partial y}\left(y_{0}\right) \overline{\bar{Z}}\left(y_{0}\right) \frac{\partial v}{\partial y}\left(y_{0}\right)^{T} .
\end{aligned}
$$

Proof of Theorem 1.3. Let

$$
\Psi_{\varepsilon}(\tau, \omega)=\hat{\psi}\left(X_{\varepsilon}\left(\tau \varepsilon^{-4 / 3}, \omega\right), \tau \varepsilon^{-4 / 3}\right) .
$$

Since $\|\hat{2}\|<\infty,\left\{\Psi_{\varepsilon}(\tau, \omega)\right\}_{0<t \leqq \varepsilon_{0}}$ is a weakly sequentially compact set in $L_{1}\left(\left[0, \tau_{0}\right] \times \Omega, m \times P\right)$ for each $\tau_{0}$, where $m$ is Lebesgue measure [5]. Using a diagonalization argument, given any sequence of the $\Psi_{\varepsilon}$ 's, there exists a subsequence that converges weakly in $L_{1}([0, \tau] \times \Omega, m \times P)$ for every finite $\tau$. By Theorem 1.1 , the $\hat{V}_{\varepsilon}$ processes are relatively compact, and from any weakly convergent sequence of $\hat{V}_{\varepsilon}$ we can extract an $L_{1}$-weakly convergent subsequence $\hat{V}_{\varepsilon_{n}}$ such that $\Psi_{\varepsilon_{n}} \rightarrow \Psi_{0}$ for some $\Psi_{0}$, the convergence holding for $L_{1}([0, \tau] \times \Omega$, $m \times P)$ and each $\tau<\infty$. It suffices to establish that the asserted uniform limit distribution of $Z_{\varepsilon}\left(\tau \varepsilon^{-4 / 3}\right)(\bmod \xi)$ is approached by all such sequences in the iterated limit as $\varepsilon_{n} \rightarrow 0$ then $\tau \rightarrow \infty$.

Using the Skorohod representation theorem, letting $\tau_{1}<\tau_{2}<\cdots<$ $\tau_{k} \leqq \tau<\tau+\delta$ and $\eta: \mathbb{R}^{k} \rightarrow \mathbb{R}^{1}$ be bounded and continuous,

$$
\begin{aligned}
E & {\left[\eta\left(V_{0}\left(\tau_{1}\right), \ldots, V_{0}\left(\tau_{k}\right)\right)\left(V_{0}(\tau+\delta)-V_{0}(\tau)\right)^{Q}\right] } \\
& =\lim _{n \rightarrow \infty} E\left[\eta\left(\hat{V}_{\varepsilon_{n}}\left(\tau_{1}\right), \ldots, \hat{V}_{\varepsilon_{n}}\left(\tau_{k}\right)\right)\left(\hat{V}_{\varepsilon_{n}}(\tau+\delta)-\hat{V}_{\varepsilon_{n}}(\tau)\right)^{Q}\right] \\
& =\lim _{n \rightarrow \infty} E \int_{\tau}^{\tau+\delta} \eta\left(\hat{V}_{\varepsilon_{n}}\left(\tau_{1}\right), \ldots, \hat{V}_{\varepsilon_{n}}\left(\tau_{k}\right)\right) \Psi_{\varepsilon_{n}}(s) d s \\
& =\lim _{n \rightarrow \infty} E \int_{\tau}^{\tau+\delta} \eta\left(V_{0}\left(\tau_{1}\right), \ldots, V_{0}\left(\tau_{k}\right)\right) \Psi_{\varepsilon_{n}}(s) d s \\
& =E \int_{\tau}^{\tau+\delta} \eta\left(V_{0}\left(\tau_{1}\right), \ldots, V_{0}\left(\tau_{k}\right)\right) \Psi_{0}(s) d s,
\end{aligned}
$$

where at the second equality we use (3.15) and at the third equality we use the a.e. convergence of $V_{\varepsilon_{n}}\left(\tau_{j}\right)$ to $V_{0}\left(\tau_{j}\right)$, which implies

$$
\begin{aligned}
& \left|E \int_{\tau}^{\tau+\delta}\left(\eta\left(\hat{V}_{\varepsilon_{n}}\left(\tau_{1}\right), \ldots, \hat{V}_{\varepsilon_{n}}\left(\tau_{k}\right)\right)-\eta\left(V_{0}\left(\tau_{1}\right), \ldots, V_{0}\left(\tau_{k}\right)\right)\right) \Psi_{\varepsilon_{n}}(s) d s\right| \\
& \quad \leq\|\hat{4}\| \int_{\tau}^{\tau+\delta} E\left|\eta\left(\hat{V}_{\varepsilon_{n}}\left(\tau_{1}\right), \ldots, \hat{V}_{\varepsilon_{n}}\left(\tau_{k}\right)\right)-\eta\left(V_{0}\left(\tau_{1}\right), \ldots, V_{0}\left(\tau_{k}\right)\right)\right| d s \rightarrow 0,
\end{aligned}
$$


as $n \rightarrow \infty$ by the dominated convergence theorem. Again we set $\mathscr{A}_{\tau}=$ $\sigma\left(V_{0}(s), s \leqq \tau\right)$. Then (3.27) implies

$$
E\left(\left(V_{0}(\tau+\delta)-V_{0}(\tau)\right)^{Q} \mid \mathscr{A}_{\tau}\right)=E\left(\int_{\tau}^{\tau+\delta} \Psi_{0}(s) d s \mid \mathscr{A}_{\tau}\right) \text { a.s. . }
$$

Let $\Psi(\tau)=E\left(\Psi_{0}(\tau) \mid \mathscr{A}_{\tau}\right)$. Then

$$
E\left(\int_{\tau}^{\tau+\delta} \Psi_{0}(s) d s \mid \mathscr{A}_{\tau}\right)=E\left(\int_{\tau}^{\tau+\delta} E\left(\Psi_{0}(s) \mid \mathscr{A}_{s}\right) d s \mid \mathscr{A}_{\tau}\right)=E\left(\int_{\tau}^{\tau+\delta} \Psi_{0}(s) d s \mid \mathscr{A}_{\tau}\right) \text { a.s. }
$$

and

$$
\begin{gathered}
E\left(\frac{\partial v}{\partial y}\left(y_{0}\right)\left(V_{0}(\tau+\delta)-V_{0}(\tau)\right)^{Q} \frac{\partial v}{\partial y}\left(y_{0}\right)^{T} \mid \mathscr{A}_{\tau}\right) \\
=E\left(\int_{\tau}^{\tau+\delta} \frac{\partial v}{\partial y}\left(y_{0}\right) \Psi(s) \frac{\partial v}{\partial y}\left(y_{0}\right)^{T} d s \mid \mathscr{A}_{\tau}\right) \text { a.s. }
\end{gathered}
$$

As in Theorem 1.1, $\frac{\partial y}{\partial y}\left(y_{0}\right) V_{0}(\tau)$ is a martingale in $C_{q}\left[0, \tau_{0}\right]$ with $\frac{\partial y}{\partial y}\left(y_{0}\right) V_{0}(0)=0$. By $[4] \frac{\partial y}{\partial y}\left(y_{0}\right) V_{0}(\tau)$ has the representation

$$
\frac{\partial v}{\partial y}\left(y_{0}\right) V_{0}(\tau)=\int_{0}^{\tau}\left(\frac{\partial v}{\partial y}\left(y_{0}\right) \Psi(s) \frac{\partial v}{\partial y}\left(y_{0}\right)^{T}\right)^{1 / 2} d B(s),
$$

where $B(s)$ is a Brownian motion process in $\mathbb{R}^{q}$ with $B(\tau+\delta)-B(\tau)$ independent of $\mathscr{A}_{\tau}$ for each $\tau$.

Next we establish a lower bound on $\frac{\partial y}{\partial y}\left(y_{0}\right) \Psi \frac{\partial y}{\partial y}\left(y_{0}\right)^{T}$ using (A7). Let $A \in \mathscr{F}$ and $\tau, \delta \geqq 0$ and let $K=T_{1} T_{2}$ :

$$
\begin{aligned}
& \int_{A}^{\tau} \int_{\tau}^{\tau+\delta} \frac{\partial v}{\partial y}\left(y_{0}\right) \Psi_{\varepsilon}(s) \frac{\partial v}{\partial y}\left(y_{0}\right)^{T} d s d P \\
& =\int_{A}^{\tau} \int_{\tau}^{\tau+\delta} \frac{1}{T_{1}} \int_{0}^{T_{1}} \frac{\partial v}{\partial y}\left(y_{0}\right) \hat{\psi}\left(X_{\varepsilon}\left(s \varepsilon^{-4 / 3}-t\right), s \varepsilon^{-4 / 3}\right) \frac{\partial v}{\partial y}\left(y_{0}\right)^{T} d t d s d P+O(\varepsilon) \\
& =\int_{A}^{\tau} \int_{\tau}^{\tau+\delta} \frac{1}{T_{1}} \int_{0}^{T_{1}} \frac{\partial v}{\partial y}\left(y_{0}\right) \hat{\psi}\left(X_{\varepsilon}\left(s \varepsilon^{-4 / 3}\right), s \varepsilon^{-4 / 3}+t\right) \frac{\partial v}{\partial y}\left(y_{0}\right)^{T} d t d s d P+O(\varepsilon) \\
& \geqq \int_{A}^{\tau} \int_{\tau}^{\tau+\delta} \frac{1}{T_{1}} \Delta\left(X_{\varepsilon}\left(s \varepsilon^{-4 / 3}\right)\right) d s d P+O(\varepsilon)
\end{aligned}
$$

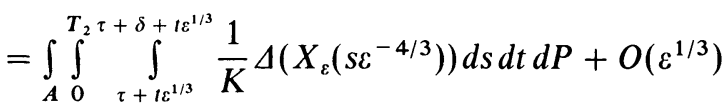

$$
\begin{aligned}
& =\int_{A} \int_{0}^{T_{2} \tau} \int_{\tau}^{\tau+\delta} \frac{1}{K} \Delta\left(X_{\varepsilon}\left(s \varepsilon^{-4 / 3}+t \varepsilon^{-1}\right)\right) d s d t d P+O\left(\varepsilon^{1 / 3}\right) \\
& =\int_{A}^{\tau} \int_{\tau}^{\tau+\delta} \int_{0}^{T_{2}} \frac{1}{K} \Delta\left(X_{\varepsilon}\left(s \varepsilon^{-4 / 3}+t \varepsilon^{-1}\right)\right) d s d t d P+O\left(\varepsilon^{1 / 3}\right) \\
& =\int_{A}^{\tau} \int_{\tau}^{\tau+\delta} \int_{0}^{T_{2}} \frac{1}{K} \Delta\left(\bar{x}\left(t, X_{\varepsilon}\left(s \varepsilon^{-4 / 3}\right)\right)\right) d s d t d P+o(1) \\
& \geqq \int_{A}^{\tau} \int_{\tau}^{\tau+\delta} \frac{1}{K} \Delta_{0} d s d P+o(1)
\end{aligned}
$$

where Proposition 3.3 is applied at the next to last line. 
Thus

$$
\int_{A}^{\tau} \int_{\tau}^{\tau} \frac{\partial v}{\partial y}\left(y_{0}\right) \Psi_{\varepsilon}(s) \frac{\partial v}{\partial y}\left(y_{0}\right)^{T} d s d P \geqq \int_{A}^{\tau} \int_{\tau}^{+\delta} \frac{1}{K} \Delta_{0} d s d P
$$

and it follows that $\frac{\partial v}{\partial y}\left(y_{0}\right) \Psi_{0} \frac{\partial v}{\partial y}\left(y_{0}\right)^{T} \geqq K^{-1} \Delta_{0}$ a.e. and, since conditional expectation is a positive operator, $\frac{\partial y}{\partial y}\left(y_{0}\right) \Psi \frac{\partial y}{\partial y}\left(y_{0}\right)^{T} \geqq K^{-1} \Delta_{0}$ a.e. Let $B^{(1)}$ be Brownian motion in $\mathbb{R}^{q}$ with $B^{(1)}(\tau+\delta)-B^{(1)}(\tau)$ independent of $\mathscr{A}_{\tau}$, for each $\tau$, and let $B^{(2)}$ be a second Brownian motion in $\mathbb{R}^{q}$ independent of $B^{(1)}$ and $\sigma\left(\cup \mathscr{A}_{\tau}\right)$. Then

$$
M(\tau):=\int_{0}^{\tau}\left(\frac{\partial v}{\partial y}\left(y_{0}\right) \Psi(s) \frac{\partial v}{\partial y}\left(y_{0}\right)^{T}-\frac{1}{K} \Delta_{0}\right)^{1 / 2} d B^{(1)}(s)+\left(\frac{1}{K} \Delta_{0}\right)^{1 / 2} B^{(2)}(\tau),
$$

is a martingale with respect to $\mathscr{B}_{\tau}:=\sigma\left(\mathscr{A}_{\tau} \cup \sigma(M(s), 0 \leqq s \leqq \tau)\right)$ and satisfies

$$
E\left((M(\tau+\delta)-M(\tau))^{Q} \mid \mathscr{B}_{\tau}\right)=E\left(\int_{\tau}^{\tau+\delta} \frac{\partial v}{\partial y}\left(y_{0}\right) \Psi(s) \frac{\partial v}{\partial y}\left(y_{0}\right)^{T} d s \mid \mathscr{B}_{\tau}\right) \text { a.s. }
$$

But then $M(\tau)$ has a representation like that in (3.28) with $\mathscr{A}_{\tau}$ replaced by $\mathscr{B}_{\tau}$, so this process is equal in distribution to the process $\frac{\partial y}{\partial y}\left(y_{0}\right) V_{0}(\tau)$. Using (3.26), $W_{0}(\tau)$ is equal in distribution to

$$
\int_{0}^{\tau} \int_{0}^{s}\left(\frac{\partial v}{\partial y}\left(y_{0}\right) \Psi(s) \frac{\partial v}{\partial y}\left(y_{0}\right)^{T}-\frac{1}{K} \Delta_{0}\right)^{1 / 2} d B^{(1)}(t) d s+\left(\frac{1}{K} \Delta_{0}\right)^{1 / 2} \int_{0}^{\tau} B^{(2)}(s) d s .
$$

The second term is independent of the first and Gaussian with covariance matrix $\left(\tau^{3} / 3 K\right) \Delta_{0}$. The density for $W_{0}(\tau)$ is the convolution of this Gaussian density with that of the first term, and has derivative bounded by the derivative of the Gaussian density. It follows that $W_{0}(\tau)(\bmod \zeta)$ is approximately uniform for large $\tau$.

\section{References}

1. Billingsley, P.: Convergence of Probability Measures, New York: Wiley, 1968

2. Borodin, A.N.: A limit theorem for solutions of differential equations with random right-hand side. Theory Prob. Appl. 22, 482-497 (1977)

3. Cogburn, R., Ellison, J.A.: A stochastic theory of adiabatic invariance. Commun. Math. Phys. 148, 97-126 (1992)

4. Doob, J.L.: Stochastic Processes. New York: Wiley, 1953

5. Dunford, N., Schwartz, J.: Linear Operators Part I: General Theory. New York: WileyInterscience, 1958

6. Ellison, J.A., Guinn, T.: Statistical equilibrium, planar channeling and the continuum model. Phys. Rev. B 13, 1880-1883 (1976)

7. Ethier, S.N., Kurtz, T.G.: Markov Processes: Characterization and Convergence. New York: Wiley-Interscience, 1986

8. Freidlin, M.I., Wentzell, A.D.: Random Perturbations of Dynamical Systems. Berlin, Heidelberg, New York: Springer, 1984

9. Khas'minskii, R.Z.: On processes defined by differential equations with a small parameter. Theory Prob. Appl. 11, 211-228 (1966)

10. Khas'minskii, R.Z.: A limit theorem for solutions of differential equations with random right-hand side. Theory Prob. Appl. 11, 390-406 (1966) 